\title{
Introduction \\ Probing the limits of regional tissue oxygenation measures
} Michael R Pinsky ${ }^{1}$ and Didier Payen ${ }^{2}$

\author{
${ }^{1}$ Professor of Critical Care Medicine, Bioengineering, Cardiovascular Disease and Anesthesiology, 606 Scaife Hall, 3550 Terrace Street, Pittsburgh, \\ PA 15261, USA \\ ${ }^{2}$ Department of Anesthesiology \& Critical Care, Hôpital Lariboisière, AP-HP, Université Paris 7 Denis Diderot, 2 rue Ambroise Paré, 75010 Paris, \\ France
}

Corresponding author: Didier Payen, dpayen1234@aol.com

Published: 30 November 2009

This article is online at http://ccforum.com/content/13/S5/S1

(c) 2009 BioMed Central Ltd

Critical Care 2009, 13(Suppl 5):S1 (doi:10.1186/cc7999)

Assessment of tissue health is the Holy Grail in determining whether patients are in shock and when resuscitation efforts can be stopped because recovery has occurred. Tissue health, itself, results from the complex interaction between cardiac pump function, circulating blood volume and vasomotor tone, the number of capillary units perfused, and the ability of the tissue to use the delivered oxygen. Although the macrocirculation seems well evaluated both invasively and noninvasively using measures of blood pressure, cardiac output and mixed venous oxygen saturation, some studies suggest that resuscitation aiming only towards macrocirculatory parameters might be insufficient to improve tissue perfusion.

Presently, there are neither generally accepted measures of global tissue viability nor reasonable leading candidate measures on the horizon. For example, although the strong ion gap assessment of metabolic acidosis predicts mortality in trauma patients, it cannot be used to assess response to therapy because crystalloid resuscitation itself alters the strong ion gap [1]. Furthermore, although regional measures of metabolism such as the sublingual tissue partial pressure of carbon dioxide [2] and urethral mucosal NAD/NADH ratios [3] track circulatory deficiency and resuscitation, their ability to assess the global status and their intrinsically highly unstable baseline values make their routine clinical use limited.

Non-invasive continuous measurements of tissue hemoglobin oxygenation using near-infrared spectroscopy have been studied and proposed to assess tissue hypoperfusion. The absolute tissue oxygen saturation $\left(\mathrm{StO}_{2}\right)$ has been proven to be instrumental in predicting outcome in trauma patients [4] but is otherwise relatively insensitive to define earlier stages of tissue hypoperfusion, despite adequate macrocirculation resuscitation aiming to prevent ischemic organ injury. The use of continuous $\mathrm{StO}_{2}$ as a marker of tissue perfusion is still being studied, however, and potentially may demonstrate greater sensitivity early on in trauma resuscitation.

Increased interest in functional hemodynamic monitoring [5], for which the fluid challenge or exercise stress test are classic examples, has recently been applied since observed static numbers cannot really evaluate the adequacy of tissue perfusion, especially regional measures of blood flow and tissue viability. Although systemic hypotension with hypoperfusion, a low mixed venous oxygen saturation and elevated serum lactate levels may be used to identify tissue ischemia, normal blood pressure, flow rates, mixed venous oxygen saturation and lactate levels do not ensure the adequacy of resuscitation. In this regard, the use of a transient vascular occlusion test (VOT) to assess $\mathrm{StO}_{2}$ responses has gained increasing interest.

The use of an ischemic challenge, or VOT, has further improved and expanded its predictive ability to scenarios such as trauma, severe sepsis and septic shock [6-8]. The VOT is a provocative test in which $\mathrm{StO}_{2}$ is measured on a distal site, such as the thenar eminence, whilst a transient rapid vascular occlusion is performed, using a sphygmomanometer, for either a defined time interval (for example, 3 minutes) or until the $\mathrm{StO}_{2}$ decreases to a defined minimal threshold. Following this, the vascular occlusion is released and the $\mathrm{StO}_{2}$ is allowed to recover. Several emergent parameters arise from this technique, including the rate of deoxygenation (thought to reflect the local metabolic rate), the rate of reoxygenation (thought to reflect local cardiovascular reserve and microcirculatory flow), and the postobstructive hyperemic response. The VOT has not been standardized, however, and has to be better characterized before making a decision on its relevance.

$\mathrm{StO}_{2}=$ absolute tissue oxygen saturation; $\mathrm{VOT}=$ vascular occlusion test. 
Specifically, $\mathrm{StO}_{2}$ can be measured at different anatomical sites (thenar, forearm, deltoid), using different near-infrared spectroscopy probe sizes (15 mm vs. $25 \mathrm{~mm}$ ) and different types and levels of ischemic deflation thresholds or ischemic times. Information is reported in the present issue on methodological issues such as depth for measurement, different modes of VOT, and determinants of VOT abnormalities. Finally, the $\mathrm{StO}_{2}$ behavior in different disease states and forms of resuscitation and the associate VOT parameters have not been defined. Given the non-invasive and potentially automated nature of $\mathrm{StO}_{2}$ and the VOT, respectively, if these measures are proven useful in clinical decision-making, then their impact on overall resuscitation management would be great.

The present supplement to Critical Care aims to address several aspects of these issues, from defining the basic physics of near-infrared spectroscopy and the VOT to clinical studies further exploring the potential of this technique in different patient groups. As with all new technologies applied to the clinical sphere, it is mandatory to identify their operating characteristics and limitations before extending their measures to patient care. Following that, it is always useful to remember that no monitoring device - no matter how insightful its data - will improve patient outcome unless coupled with a treatment that improves outcome.

\section{Competing interests}

MRP is consultant to Hutchinson Industries regarding the clinical applications of the InSpectra probe, has received honoraria for lectures at scientific symposia, and has received a grant to study $\mathrm{StO}_{2}$ in a prospective clinical trial. DP is a consultant to Hutchinson Industries regarding clinical application, received honoraria for lectures at scientific symposia, and grants for the study of $\mathrm{StO}_{2}$ in diferent patient populations, and lifethreatening conditions.

\section{Acknowledgement}

This article is part of Critical Care Volume 13 Supplement 5: Tissue oxygenation $\left(\mathrm{StO}_{2}\right)$ in healthy volunteers and critically-ill patients. The full contents of the supplement are available online at http://ccforum. com/supplements/13/S5. Publication of the supplement has been supported with funding from Hutchinson Technology Inc.

\section{References}

1. Kaplan LJ, Kellum JA: Initial pH, base deficit, lactate, anion gap, strong ion difference, and strong ion gap predict outcome from major vascular injury. Crit Care Med 2004, 32:11201124.

2. Nakagawa $Y$, Weil MH, Tang $W$, Sun $S$, Yamaguchi $H$, Jin $X$, Bisera J: Sublingual capnometry for diagnosis and quantitation of circulatory shock. Am J Respir Crit Care Med 1998, 157: 1838-1843.

3. Clavijo JA, van Bastelaar J, Pinsky MR, Puyana JC, Mayevsky A: Minimally invasive real time monitoring of mitochondrial NADH and tissue blood flow in the urethral wall during hemorrhage and resuscitation. Med Sci Monit 2008, 14:BR175BR182.

4. Cohn SM, Nathens AB, Moore FA, Rhee P, Puyana JC, Moore EE, Beilman GJ: Tissue oxygen saturation predicts the development of organ dysfunction during traumatic shock resuscitation. J Trauma 2007, 62:44-54.
5. Pinsky MR, Payen D: Functional hemodynamic monitoring. Crit Care 2005, 9:566-572.

6. Gomez H, Torres A, Polanco P, Kim HK, Zenker S, Puyana JC, Pinsky MR: Use of non-invasive NIRS during a vascular occlusion test to assess dynamic tissue $\mathrm{O}_{2}$ saturation response. Intensive Care Medicine 2008, 34:1600-1607.

7. Creteur J, Carollo T, Soldati G, Buchele G, De Backer D, Vincent JL: The prognostic value of muscle $\mathrm{StO}_{2}$ in septic patients. Intensive Care Med 200, 733:1549-1556.

8. Convertino VA, Ryan KL, Rickards CA, Salinas J, McManus JG, Cooke WH, Holcomb JB: Physiological and medical monitoring for en route care of combat casualties. J Trauma 2008, 64: S342-S353. 